\title{
Nuclear excitations as coupled one and two random-phase-approximation modes
}

\author{
D. Gambacurta, ${ }^{1,2}$ F. Catara, ${ }^{2,3}$ M. Grasso, ${ }^{4}$ M. Sambataro, ${ }^{2}$ M. V. Andrés,${ }^{5}$ and E. G. Lanza ${ }^{2,3}$ \\ ${ }^{1}$ ELI-NP, Horia Hulubei National Institute for R\&D in Physics and Nuclear Engineering, 077125 Magurele, Romania \\ ${ }^{2}$ INFN-Sezione di Catania, 95123 Catania, Italy \\ ${ }^{3}$ Dipartimento di Fisica e Astronomia, Universitá di Catania, Italy \\ ${ }^{4}$ Institut de Physique Nucléaire, IN2P3-CNRS, Université Paris-Sud, Université Paris-Saclay, F-91406 Orsay Cedex, France \\ ${ }^{5}$ Departamento de Física Atómica, Molecular y Nuclear, Universidad de Sevilla, Apdo. 1065, E-41080 Sevilla, Spain
}

(Received 29 September 2015; revised manuscript received 12 January 2016; published 9 February 2016)

\begin{abstract}
We present an extension of the random-phase approximation (RPA) where the RPA phonons are used as building blocks to construct the excited states. In our model, that we call double RPA (DRPA), we include up to two RPA phonons. This is an approximate and simplified way, with respect to the full second random-phase approximation (SRPA), to extend the RPA by including two-particle-two-hole configurations. Some limitations of the standard SRPA model, related to the violation of the stability condition, are not encountered in the DRPA. We also verify in this work that the energy-weighted sum rules are satisfied. The DRPA is applied to low-energy modes and giant resonances in the nucleus ${ }^{16} \mathrm{O}$. We show that the model (i) produces a global downwards shift of the energies with respect to the RPA spectra and (ii) provides a shift that is, however, strongly reduced compared to that generated by the standard SRPA. This model represents an alternative way of correcting for the SRPA anomalous energy shift, compared to a recently developed extension of the SRPA, where a subtraction procedure is applied. The DRPA provides results in good agreement with the experimental energies, with the exception of those low-lying states that have a dominant two-particle-two-hole nature. For describing such states, higher-order calculations are needed.
\end{abstract}

DOI: 10.1103/PhysRevC.93.024309

\section{INTRODUCTION}

A common feature of many-body systems is the presence of collective modes in their excitation spectra. Particularly interesting are those which can be interpreted in terms of vibrations. In nuclei, they have been known for a long time [1] and are observed both in the low-lying and higher-energy region (giant resonances (GR)) [1,2]. Another example of collective vibrations is the dipole plasmon resonance in metallic clusters $[3,4]$, a very collective mode which is the analog of the nuclear dipole GR and is due to the vibration of the center of mass of the delocalized valence electrons against that of the positive ions.

In all these cases, several characteristic properties are rather well reproduced within the random-phase approximation (RPA), which is for this reason considered a good microscopic theory for the study of collective states in manybody systems $[5,6]$. Among its merits, we mention that the RPA preserves the energy-weighted sum rules (EWSR), in the sense of the Thouless theorem [7]. This feature is very important because it guarantees that spurious states associated with broken symmetries are exactly separated in RPA from the physical states of the system.

On the other hand, the RPA model has some limits. Among them we recall that, by construction, it predicts a perfectly harmonic spectrum and cannot reproduce the spreading width of the excited modes. As underlined in several studies, the coupling of one-particle-one-hole (1p-1h) configurations with more complex states is very important to overcome these limitations and to describe the spreading widths and the anharmonicities in the excitation spectra (see, for example, Refs. [8-10] and references therein). A natural extension of the
RPA is the so-called second RPA (SRPA), which amounts to enlarge the space of basic elementary excitations by including two-particle-two-hole ( $2 \mathrm{p}-2 \mathrm{~h}$ ) configurations and by coupling them with the $1 \mathrm{p}-1 \mathrm{~h}$ ones and among themselves. It was shown [11-13] that the EWSR are exactly the same in SRPA and in RPA.

Recent applications of the SRPA model were performed without any cut and approximations in the matrices. Such applications provided a very strong modification (a large downwards energy shift) of the RPA excitation spectra, also for those collective states whose description within the RPA is rather good. This was obtained for collective nuclear excitations with Skyrme and Gogny forces [14,15], as well as with an interaction derived from the Argonne V18 nucleon-nucleon potential with the unitary correlation operator method [16]. In the case of metallic clusters, within the jellium model [4], the modifications found when passing from RPA to SRPA are very large [17] and completely spoil the quality of the RPA results concerning the dipole strength distribution. In this application, the limiting case of highly ionized clusters is very striking. The RPA is able to reproduce the exact theoretical prediction on the position of the dipole plasmon excitation (at the Mie energy) and the fact that the dipole strength is totally concentrated at this energy [18]. These very nice features are completely lost in SRPA [17]. In the nuclear case, one might associate such unpleasant result to some inadequacy of the used nuclear interaction. This could be especially true for the Skyrme- and Gogny-based SRPA calculations because some matrix elements appearing in SRPA are not present at the RPA level, for instance, those with three-particle and one-hole indexes or three-hole and one-particle indexes. Therefore, they do not enter at all in the fitting procedure used to fix the 
parameters of the effective interaction. Furthermore, a certain amount of correlations is already contained in an effective way in the parameters of these phenomenological forces, because the adjustment of the parameters is performed with mean-field calculations. When correlations are explicitly introduced in models beyond the mean field, double-counting problems may arise. Finally, Skyrme and Gogny forces contain zero-range terms and this produces in some cases a cutoff dependence in beyond-mean-field models.

However, the problem of the large downwards energy shift exists also in the case of metallic clusters [17] where there is no cutoff dependence and the Coulomb interaction does not contain any parameter [19]. The origin of the problem must then be different.

In deriving the equations of motion in SRPA $[11,20]$ use is made, as in RPA, of the quasiboson approximation (QBA) and it has been argued [21-23] that its use in SRPA is even more questionable than in RPA. Recently, this was more precisely understood as related to the replacement of the correlated ground state with the Hartree-Fock (HF) one, that is generated by the use of the QBA. This replacement produces a violation of the stability condition at the SRPA level [13]. A careful analysis of the merits and limits of the SRPA was presented in Ref. [13]. In particular, the violation of the stability condition in SRPA [24] is illustrated and a generalization of the Thouless theorem [7] is proven in the case where a correlated ground state is used. Very recently, a subtraction procedure able to remove the double counting in beyond-mean-field theories based on effective interactions [25,26] was applied to the SRPA case [27]. Reference [26] showed explicitly that this subtraction method is also able of ensuring the stability condition in extended RPA models such as the SRPA. In Ref. [27], very encouraging results were found for both low-lying and higher-energy excitations. In particular, the strong SRPA downward shift is corrected for those states having a dominant $1 \mathrm{p}-1 \mathrm{~h}$ nature such as the giant resonances, and the found results are in much better agreement with the experimental ones, compared to the standard SRPA results.

In Ref. [28], an extension of the SRPA including correlations in the ground state was tested by applying it to a solvable model and by comparing the results with the exact ones. It was also applied to metallic clusters [29], finding much better results than in SRPA. However, such an extended SRPA is very demanding from a computational point of view and its application to the study of atomic nuclei is hardly feasible.

On the other hand, approaches like the particle-vibration coupling [30], where one uncorrelated particle-hole configuration is coupled to a correlated collective mode, do not suffer from the violation of the stability condition $[7,13]$ encountered in SRPA. They are, however, also affected by the cutoff dependence and the double-counting problems if phenomenological zero-range interactions are employed.

Extensions of RPA including multiphonon excitations have been applied in several contexts. They make use of mapping procedures to replace the fermion particle-hole operators by their boson images [31]. The limits of such procedure were tested in Ref. [32] within a solvable model, by comparing the exact results with those obtained by truncating the mapping at different levels. It was shown that in order to correct for the violations of the Pauli principle, higher-order terms are required in the boson expansion. This makes the application of the approach to realistic systems not feasible. The procedure presented in Refs. $[33,34]$ allows one to enlarge iteratively the space in which the Hamiltonian is diagonalized by including multiphonon states built by Tamm-Dancoff phonon operators remaining in the fermion space.

The approach that we are going to present consists in considering the RPA collective fermion operators as building blocks to construct the space of states by acting repeatedly on the RPA ground state (that is, the vacuum of the RPA operators). The Hamiltonian is then diagonalized in such space. Since the fermionic structure of the collective elementary excitations is fully accounted for, one may expect to get satisfactory results with a basis of states containing up to two such elementary excitations. Thus, one considers, as in SRPA, a space containing $1 \mathrm{p}-1 \mathrm{~h}$ and $2 \mathrm{p}-2 \mathrm{~h}$ configurations, the latter appearing only through the two RPA phonon states. Since the $2 \mathrm{p}-2 \mathrm{~h}$ sector of the basis space is built with those collective excitations which already contain some effects of the residual interaction, one might envisage reducing the dimensions of the space by including only some configurations selected, for example, on the basis of their collectivity. When studying the excitation spectrum, one may introduce an energy cutoff and consider only those basis vectors whose RPA energy is lower. This would allow one to reduce the heavy computational effort often required within the SRPA. This approach is very much related to that of Ref. [35]. We stress that we do not perform here a diagonalization in the multiphonon space, but we solve generalized equations of motion. Full self-consistency is achieved by employing the same Skyrme interaction at the RPA level and for the solution of such equations of motion. We consider here RPA operators acting on the correlated RPA ground state, which is differentthan what is done in Refs. [33,34], where Tamm-Dancoff phonons were used to construct the basis states by acting on the uncorrelated HF ground state. Therefore, even considering the mixing of configurations containing up to two phonons, we take into account higher-order excitations. We call our model double RPA and use in what follows the acronym DRPA to denote it.

The approach is described in the next section. The energyweighted sum rules are shown to be satisfied in Sec. III, and applications to the nucleus ${ }^{16} \mathrm{O}$ are presented in Sec. IV. Finally, in Sec. V we draw some conclusions.

\section{THE DRPA MODEL}

In RPA one defines the operators

$$
Q_{v}^{\dagger}=\sum_{p h}\left(X_{p h}^{v} b_{p h}^{\dagger}-Y_{p h}^{v} b_{p h}\right)
$$

and $Q_{\nu}$, such that

$$
Q_{\nu}\left|\Psi_{0}(R P A)\right\rangle=0
$$

and

$$
\left|\Psi_{\nu}(R P A)\right\rangle=Q_{\nu}^{\dagger}\left|\Psi_{0}(R P A)\right\rangle,
$$

where $\left|\Psi_{0}(R P A)\right\rangle$ and $\left|\Psi_{\nu}(R P A)\right\rangle$ are the RPA ground and excited states of the system, respectively. The operators $b_{p h}^{\dagger}$ 
and $b_{p h}$ create and annihilate, respectively, a particle-hole pair $\left(b_{p h}^{\dagger}=a_{p}^{\dagger} a_{h}\right)$. Here and in the following, coupling to total quantum numbers is understood. The $X$ and $Y$ amplitudes appearing in Eq. (1) are solutions of the equations of motion

$$
\left(\begin{array}{ll}
A^{11} & B^{11} \\
A^{* 11} & B^{* 11}
\end{array}\right)\left(\begin{array}{l}
X^{v} \\
Y^{\nu}
\end{array}\right)=E_{\nu}\left(\begin{array}{cc}
G^{11} & 0 \\
0 & -G^{* 11}
\end{array}\right)\left(\begin{array}{l}
X^{v} \\
Y^{\nu}
\end{array}\right),
$$

where $[5,6]$

$$
\begin{aligned}
A_{p h, p^{\prime} h^{\prime}}^{11} & =\left\langle\Psi_{0}(R P A)\left|\left[b_{p h},\left[H, b_{p^{\prime}, h^{\prime}}^{\dagger}\right]\right]\right| \Psi_{0}(R P A)\right\rangle \\
& \simeq\left\langle H F\left|\left[b_{p h},\left[H, b_{p^{\prime}, h^{\prime}}^{\dagger}\right]\right]\right| H F\right\rangle, \\
B_{p h, p^{\prime} h^{\prime}}^{11} & =-\left\langle\Psi_{0}(R P A)\left|\left[b_{p h},\left[H, b_{p^{\prime}, h^{\prime}}\right]\right]\right| \Psi_{0}(R P A)\right\rangle \\
& \simeq-\left\langle H F\left|\left[b_{p h},\left[H, b_{p^{\prime}, h^{\prime}}\right]\right]\right| H F\right\rangle,
\end{aligned}
$$

and

$$
\begin{aligned}
G_{p h, p^{\prime} h^{\prime}}^{11} & =\left\langle\Psi_{0}(R P A)\left|\left[b_{p h}, b_{p^{\prime}, h^{\prime}}^{\dagger}\right]\right| \Psi_{0}(R P A)\right\rangle \\
& \simeq\left\langle H F\left|\left[b_{p h}, b_{p^{\prime}, h^{\prime}}^{\dagger}\right]\right| H F\right\rangle=\delta_{p p^{\prime}} \delta_{h h^{\prime}} .
\end{aligned}
$$

As explicitly shown, in order to calculate the matrix elements appearing in Eq. (4), use is made of the QBA, replacing the RPA ground state $\left(\left|\Psi_{0}(R P A)\right\rangle\right)$ with the HF one $(|H F\rangle)$. to

In SRPA, the excitation phonon operators are generalized

$$
\begin{aligned}
\mathcal{Q}_{\rho}^{\dagger}= & \sum_{p h}\left(X_{p h}^{\rho} b_{p h}^{\dagger}-Y_{p h}^{\rho} b_{p h}\right) \\
& +\sum_{\substack{p_{1} h_{1} \\
p_{2} h_{2}}}\left(X_{p_{1} h_{1} p_{2} h_{2}}^{\rho} b_{p_{1} h_{1}}^{\dagger} b_{p_{2} h_{2}}^{\dagger}-Y_{p_{1} h_{1} p_{2} h_{2}}^{\rho} b_{p_{2} h_{2}} b_{p_{1} h_{1}}\right)
\end{aligned}
$$

which contain $2 \mathrm{p}-2 \mathrm{~h}$ components in addition to the $1 \mathrm{p}-1 \mathrm{~h}$ ones. This extension allows one to go beyond the harmonic approximation of the RPA and to describe the spreading width of the excited states through the coupling of the $1 \mathrm{p}-1 \mathrm{~h}$ configurations with the $2 \mathrm{p}-2 \mathrm{~h}$ ones. As in RPA, one assumes

$$
\left|\Psi_{\rho}(S R P A)\right\rangle=\mathcal{Q}_{\rho}^{\dagger}\left|\Psi_{0}(S R P A)\right\rangle
$$

and

$$
\mathcal{Q}_{\rho}\left|\Psi_{0}(\operatorname{SRP} A)\right\rangle=0,
$$

where $\left|\Psi_{\rho}(S R P A)\right\rangle$ and $\left|\Psi_{0}(S R P A)\right\rangle$ are the SRPA excited and ground states, respectively. The amplitudes appearing in Eq. (8) and the energies $E_{\rho}$ of the excited states are solutions of

$$
\left(\begin{array}{cc}
\mathcal{A} & \mathcal{B} \\
\mathcal{B}^{*} & \mathcal{A}^{*}
\end{array}\right)\left(\begin{array}{l}
\mathcal{X}^{\rho} \\
\mathcal{Y}^{\rho}
\end{array}\right)=E_{\rho}\left(\begin{array}{cc}
\mathcal{G} & 0 \\
0 & -\mathcal{G}^{*}
\end{array}\right)\left(\begin{array}{l}
\mathcal{X}^{\rho} \\
\mathcal{Y}^{\rho}
\end{array}\right)
$$

where

$$
\mathcal{X}_{\rho}=\left(\begin{array}{c}
X_{p h}^{\rho} \\
X_{p_{1} h_{1} p_{2} h_{2}}^{\rho}
\end{array}\right), \quad \mathcal{Y}_{\rho}=\left(\begin{array}{c}
Y_{p h}^{\rho} \\
Y_{p_{1} h_{1} p_{2} h_{2}}^{\rho}
\end{array}\right)
$$

and

$$
\mathcal{A}=\left(\begin{array}{cc}
A_{p h, p^{\prime} h^{\prime}}^{11} & A_{p h, p_{1}^{\prime} h_{1}^{\prime} p_{2}^{\prime} h_{2}^{\prime}}^{12} \\
A_{p_{1} h_{1} p_{2} h_{2}, p^{\prime} h^{\prime}}^{21} & A_{p_{1} h_{1} p_{2} h_{2}, p_{1}^{\prime} h_{1}^{\prime} p_{2}^{\prime} h_{2}^{\prime}}^{22}
\end{array}\right) .
$$

Similar expressions may be written for the (super)matrices $\mathcal{B}$ and $\mathcal{G}$. Again, in order to calculate the above matrix elements, one makes use of the QBA, $\left|\Psi_{o}(S R P A)\right\rangle \simeq|H F\rangle$. The submatrices $A^{11}, B^{11}$, and $G^{11}$ are the same as the corresponding RPA matrices. The expressions for $A^{12}, A^{21}, A^{22}$, and $G^{22}$ are analogous, except for the presence of $2 \mathrm{p}-2 \mathrm{~h}$ operators. Finally, $B^{12}=B^{21}=B^{22}=G^{12}=G^{21}=0$ for density-independent interactions or if one neglects the rearrangement terms generated by the density dependence of the interaction [36]. As said above, complete SRPA calculations (that is, including the coupling of the $1 \mathrm{p}-1 \mathrm{~h}$ configurations with the $2 \mathrm{p}-2 \mathrm{~h}$ ones and of the $2 \mathrm{p}-2 \mathrm{~h}$ among themselves) made for several systems and with different residual interactions give disturbing results that are corrected when the stability condition is ensured [27]. This may be, for example, guaranteed either by a subtraction method [27] or by using a correlated ground state [13], that is, by avoiding the QBA.

In the DRPA, the RPA collective excitations are used as building blocks and we write the excited states as

$$
\left|\Psi_{\alpha}\right\rangle=\left[\sum_{\nu} c_{\nu}^{\alpha} Q_{\nu}^{\dagger}+\sum_{\nu_{1} \leqslant \nu_{2}} d_{\nu_{1} \nu_{2}}^{\alpha} Q_{\nu_{1}}^{\dagger} Q_{v_{2}}^{\dagger}\right]\left|\Psi_{0}\right\rangle \equiv \Lambda_{\alpha}^{\dagger}\left|\Psi_{0}\right\rangle
$$

maintaining fixed the structure of the RPA operators $Q_{\nu}^{\dagger}$. If we further make the approximation

$$
\left|\Psi_{0}\right\rangle \simeq\left|\Psi_{0}(R P A)\right\rangle
$$

then it is true that

$$
\Lambda_{\alpha}\left|\Psi_{0}\right\rangle=0
$$

and

$$
\left\langle\Psi_{\alpha} \mid \Psi_{0}\right\rangle=\left\langle\Psi_{0}\left|\Lambda_{\alpha}\right| \Psi_{0}\right\rangle=0
$$

that is, the ground state $\left|\Psi_{0}\right\rangle$ is orthogonal to the excited states. We remark that, once the approximation (15) is made, the vacuum condition, Eq. (16), follows immediately from Eq. (2), satisfied by the elementary operators $Q_{v}$ at the RPA level. By following the same procedure used to derive the RPA equations of motion $[5,6]$ one gets

$$
\begin{aligned}
H\left|\Psi_{\alpha}\right\rangle & =E_{\alpha}\left|\Psi_{\alpha}\right\rangle \Rightarrow \\
{\left[H, \Lambda_{\alpha}^{\dagger}\right]\left|\Psi_{0}\right\rangle } & =\left(E_{\alpha}-E_{0}\right) \Lambda_{\alpha}^{\dagger}\left|\Psi_{0}\right\rangle
\end{aligned}
$$

From Eq. (15) and from the vacuum condition, Eq. (2), it follows

$$
\begin{aligned}
& \sum_{v} c_{v}^{\alpha}\left\langle\Psi_{0}\left|\left[Q_{v^{\prime}},\left[H, Q_{v}^{\dagger}\right]\right]\right| \Psi_{0}\right\rangle \\
& \quad+\sum_{v_{1} v_{2}} d_{v_{1} v_{2}}^{\alpha}\left\langle\Psi_{0}\left|\left[Q_{v^{\prime}},\left[H, Q_{v_{1}}^{\dagger} Q_{v_{2}}^{\dagger}\right]\right]\right| \Psi_{0}\right\rangle=\left(E_{\alpha}-E_{0}\right) c_{v^{\prime}}^{\alpha}
\end{aligned}
$$


and

$$
\begin{aligned}
\sum_{\nu} & c_{v}^{\alpha}\left\langle\Psi_{0}\left|\left[Q_{v_{2}^{\prime}} Q_{v_{1}^{\prime}},\left[H, Q_{v}^{\dagger}\right]\right]\right| \Psi_{0}\right\rangle \\
& +\sum_{v_{1} \nu_{2}} d_{v_{1} \nu_{2}}^{\alpha}\left\langle\Psi_{0}\left|\left[Q_{v_{2}^{\prime}} Q_{v_{1}^{\prime}},\left[H, Q_{v_{1}}^{\dagger} Q_{v_{2}}^{\dagger}\right]\right]\right| \Psi_{0}\right\rangle \\
= & \left(E_{\alpha}-E_{0}\right) \sum_{v_{1} v_{2}} d_{\nu_{1} v_{2}}^{\alpha}\left\langle\Psi_{0}\left|\left[Q_{v_{2}^{\prime}} Q_{v_{1}^{\prime}}, Q_{v_{1}}^{\dagger} Q_{v_{2}}^{\dagger}\right]\right| \Psi_{0}\right\rangle
\end{aligned}
$$

In order to evaluate the matrix elements appearing in the above equations we proceed exactly as in RPA: The average values in the correlated ground state are replaced by those calculated in the HF, uncorrelated, one. This is a reasonable approximation when the single-particle occupation numbers are close to 0 and 1 . We have verified that this is indeed the case.

By following this procedure Eqs (18) and (19) can be rewritten as

$$
\sum_{\nu} c_{\nu}^{\alpha} A_{\nu^{\prime} v}+\sum_{v_{1} v_{2}} d_{v_{1} \nu_{2}}^{\alpha} A_{\nu^{\prime}, v_{1} \nu_{2}}=\left(E_{\alpha}-E_{0}\right) c_{v^{\prime}}^{\alpha}
$$

and

$$
\begin{aligned}
& \sum_{\nu} c_{\nu}^{\alpha} A_{v_{2}^{\prime} v_{1}^{\prime}, v}+\sum_{v_{1} v_{2}} d_{v_{1} \nu_{2}}^{\alpha} A_{v_{2}^{\prime} v_{1}^{\prime}, v_{1} v_{2}} \\
& =\left(E_{\alpha}-E_{0}\right) \sum_{v_{1} v_{2}} d_{v_{1} \nu_{2}}^{\alpha} G_{v_{2}^{\prime} v_{1}^{\prime}, v_{1} v_{2}}
\end{aligned}
$$

with

$$
\begin{aligned}
& A_{v^{\prime} v} \equiv\left\langle H F\left|\left[Q_{v^{\prime}},\left[H, Q_{v}^{\dagger}\right]\right]\right| H F\right\rangle=E_{v}^{R P A} \delta_{v v^{\prime}}, \\
& A_{v^{\prime}, v_{1} v_{2}} \equiv\left\langle H F\left|\left[Q_{v^{\prime}},\left[H, Q_{v_{1}}^{\dagger} Q_{v_{2}}^{\dagger}\right]\right]\right| H F\right\rangle \\
& =\sum_{\substack{p^{\prime} h^{\prime} \\
p_{1} h_{1} p_{2} h_{2}}}\left(X_{p^{\prime} h^{\prime}}^{v^{\prime}} X_{p_{1} h_{1}}^{v_{1}} X_{p_{2} h_{2}}^{v_{2}}-Y_{p^{\prime} h^{\prime}}^{v^{\prime}} Y_{p_{1} h_{1}}^{\nu_{2}} Y_{p_{2} h_{2}}^{v_{1}}\right) A_{p^{\prime} h^{\prime}, p_{1} h_{1} p_{2} h_{2}}^{12} \\
& A_{v_{1}^{\prime} v_{2}^{\prime}, v}^{21}=A_{v, v_{1}^{\prime} v_{2}^{\prime}}^{12}, \\
& A_{v_{1}^{\prime} \nu_{2}^{\prime}, v_{1} \nu_{2}}^{22} \equiv\left\langle H F\left|\left[Q_{v_{2}^{\prime}} Q_{v_{1}^{\prime}},\left[H, Q_{v_{1}}^{\dagger} Q_{v_{2}}^{\dagger}\right]\right]\right| H F\right\rangle \\
& =\sum_{\substack{p_{1}^{\prime} h_{1}^{\prime} p_{2}^{\prime} h_{2}^{\prime} \\
p_{1} h_{1} p_{2} h_{2}}}\left(X_{p_{1}^{\prime} h_{1}^{\prime}}^{v_{1}^{\prime}} X_{p_{2}^{\prime} h_{2}^{\prime}}^{v_{2}^{\prime}} X_{p_{1} h_{1}}^{v_{1}} X_{p_{2} h_{2}}^{v_{2}}+Y_{p_{1} h_{1}}^{v_{1}^{\prime}} Y_{p_{2} h_{2}}^{v_{2}^{\prime}} Y_{p_{1}^{\prime} h_{1}^{\prime}}^{v_{1}} Y_{p_{2}^{\prime} h_{2}^{\prime}}^{v_{2}}\right) A_{p_{1}^{\prime} h_{1}^{\prime} p_{2}^{\prime} h_{2}^{\prime}, p_{1} h_{1} p_{2} h_{2}}^{22}+\mathcal{O}\left(B^{11}\right), \\
& G_{v_{1}^{\prime} v_{2}^{\prime}, \nu_{1} v_{2}}^{22} \equiv\left\langle H F\left|\left[Q_{v_{2}^{\prime}} Q_{v_{1}^{\prime}}, Q_{v_{1}}^{\dagger} Q_{v_{2}}^{\dagger}\right]\right| H F\right\rangle \\
& =\sum_{\substack{p_{1}^{\prime} h_{1}^{\prime} p_{2}^{\prime} h_{2}^{\prime} \\
p_{1} h_{1} p_{2} h_{2}}}\left(X_{p_{1}^{\prime} h_{1}^{\prime}}^{v_{1}^{\prime}} X_{p_{2}^{\prime} h_{2}^{\prime}}^{v_{2}^{\prime}} X_{p_{1} h_{1}}^{v_{1}} X_{p_{2} h_{2}}^{v_{2}}-Y_{p_{1}^{\prime} h_{1}^{\prime}}^{v_{1}^{\prime}} Y_{p_{2}^{\prime} h_{2}^{\prime}}^{v_{2}^{\prime}} Y_{p_{1} h_{1}}^{\nu_{1}} Y_{p_{2} h_{2}}^{\nu_{2}}\right) G_{p_{1}^{\prime} h_{1}^{\prime} p_{2}^{\prime} h_{2}^{\prime}, p_{1} h_{1} p_{2} h_{2}}^{22} \cdot
\end{aligned}
$$

The matrices in the right-hand sides of Eqs. (23)-(26) are denoted and have the same expressions as the SRPA $A^{i j}$ and $G^{22}$ matrices. The $X$ and $Y$ amplitudes are those calculated at the RPA level and contain the information on the fermionic structure of the phonons.

In Eq. (25), $\mathcal{O}\left(B^{11}\right)$ denotes several terms containing the RPA matrix $B^{11}$ of Eq. (6). In the calculations that we present in this work we neglect for simplicity the terms $\mathcal{O}\left(B^{11}\right)$ appearing in Eq. (25). We have checked within an exactly solvable threelevel Lipkin model [37] that their contribution to the DRPA energies is negligible.

\section{ENERGY-WEIGHTED SUM RULES}

It is very well known that if $\left|\Psi_{0}\right\rangle$ and $\left|\Psi_{v}\right\rangle$ are the exact eigenstates of a system with Hamiltonian $H$, the following equality holds [6]:

$$
\sum_{\nu} E_{v}\left|\left\langle\Psi_{v}|T| \Psi_{0}\right\rangle\right|^{2}=\frac{1}{2}\left\langle\Psi_{0}|[T,[H, T]]| \Psi_{0}\right\rangle
$$

with $T$ any transition operator. The above equality denotes the EWSR. The Thouless theorem [7] states that in RPA the
EWSR are valid if the sum in the left-hand side is calculated with the RPA energies and transition probabilities, whereas the uncorrelated ground state $|H F\rangle$ is used in the right-hand side. It was shown that the EWSR are valid also in SRPA [11-13]. Following step by step the demonstration of Ref. [11], one easily shows their validity also in the present approach. For any self-adjoint transition operator $T$ connecting the ground state to the excited ones we can write

$$
T=\sum_{\alpha}\left\langle\Psi_{\alpha}|T| \Psi_{0}\right\rangle \Lambda_{\alpha}^{\dagger}+\text { H.c. }
$$

and

$$
\begin{aligned}
\left\langle\Psi_{\alpha}|T| \Psi_{0}\right\rangle= & \left\langle\Psi_{0}\left|\left[\Lambda_{\alpha}, T\right]\right| \Psi_{0}\right\rangle \\
= & \sum_{\nu} c_{\nu}^{\alpha}\left\langle\Psi_{0}\left|\left[Q_{\nu}, T\right]\right| \Psi_{0}\right\rangle \\
& +\sum_{\nu, \nu^{\prime}} d_{\nu \nu^{\prime}}^{\alpha}\left\langle\Psi_{0}\left|\left[Q_{\nu} Q_{\nu^{\prime}}, T\right]\right| \Psi_{0}\right\rangle .
\end{aligned}
$$

In the spirit of the Thouless theorem the right-hand side of Eq. (27) is approximated as

$$
\left\langle\Psi_{0}|[T,[H, T]]| \Psi_{0}\right\rangle \approx\langle H F|[T,[H, T]]| H F\rangle .
$$




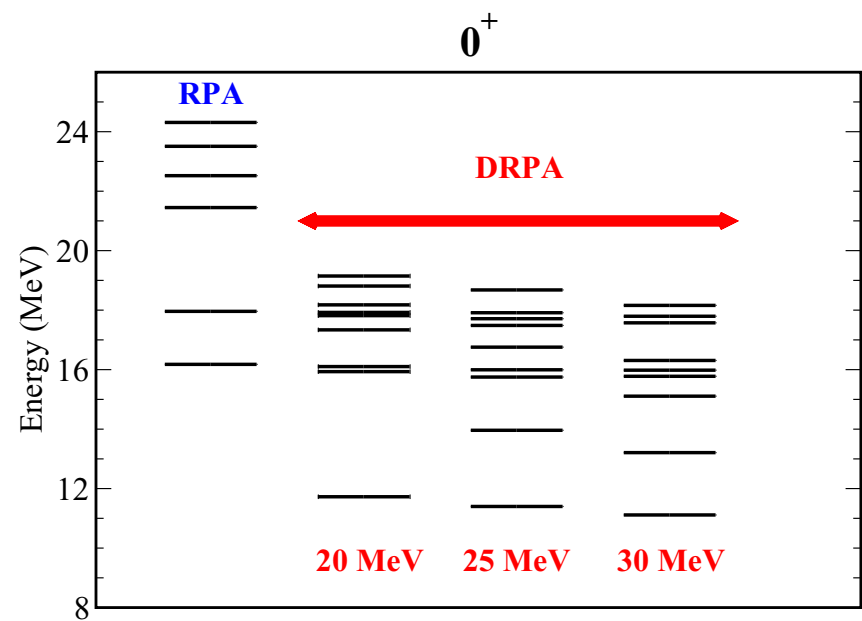

FIG. 1. Monopole states calculated with the RPA and the DRPA, with three different values of the cutoff $E 1 C$, from 20 to $30 \mathrm{MeV}$ and a value of $40 \mathrm{MeV}$ for the $E 2 C$ cutoff.

Since, as can be easily verified,

$$
\left\langle H F\left|\left[\Lambda_{\alpha}^{\dagger},\left[H, \Lambda_{\alpha}^{\dagger}\right]\right]\right| H F\right\rangle=0,
$$

one gets

$$
\begin{aligned}
& \frac{1}{2}\langle H F|[T,[H, T]]| H F\rangle \\
& =\sum_{\alpha \alpha^{\prime}} T_{0 \alpha} T_{0 \alpha^{\prime}}^{*}\left\langle H F\left|\left[\Lambda_{\alpha}^{\prime},\left[H, \Lambda_{\alpha}^{\dagger}\right]\right]\right| H F\right\rangle \\
& =\sum_{\alpha \alpha^{\prime}} T_{0 \alpha} T_{0 \alpha^{\prime}}^{*}\left[\sum_{\nu v^{\prime}} c_{\nu^{\prime}}^{\alpha^{\prime}} c_{\nu}^{\alpha} A_{\nu^{\prime} v}^{11}+\sum_{\nu^{\prime} v_{1} \nu_{2}} c_{\nu^{\prime}}^{\alpha^{\prime}} d_{v_{1} \nu_{2}}^{\alpha} A_{v^{\prime}, v_{1} \nu_{2}}^{12}\right. \\
& \left.+\sum_{\nu v_{1}^{\prime} v_{2}^{\prime}} c_{\nu}^{\alpha} d_{v_{1}^{\prime} v_{2}^{\prime}}^{\alpha^{\prime}} A_{v_{1}^{\prime} v_{2}^{\prime}, v}^{12}+\sum_{v_{1} \nu_{2} v_{1}^{\prime} v_{2}^{\prime}} d_{v_{1} v_{2}}^{\alpha} d_{v_{1}^{\prime} v_{2}^{\prime}}^{\alpha^{\prime}} A_{v_{1}^{\prime} v_{2}^{\prime}, v_{1} \nu_{2}}^{22}\right] \text {. }
\end{aligned}
$$

By acting with $\sum_{\alpha^{\prime}} T_{0 \alpha^{\prime}} \sum_{v} c_{v}^{\alpha^{\prime}} \sum_{\alpha} T_{0 \alpha}$ on the left- and right-hand sides of Eq. (18) and with $\sum_{\alpha^{\prime}} T_{0 \alpha^{\prime}} \sum_{v_{1}^{\prime} v_{2}^{\prime}}$

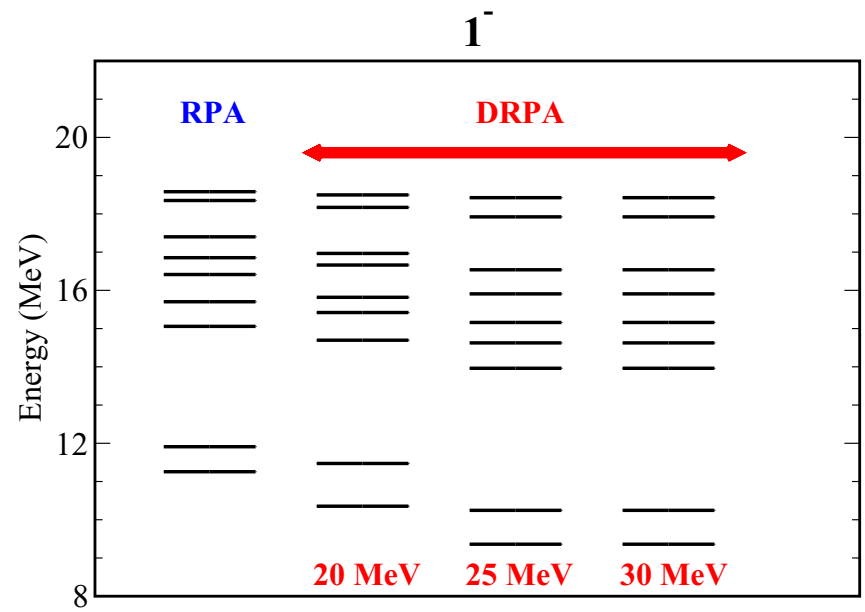

FIG. 2. Same as in Fig. 1, but for the dipole states.

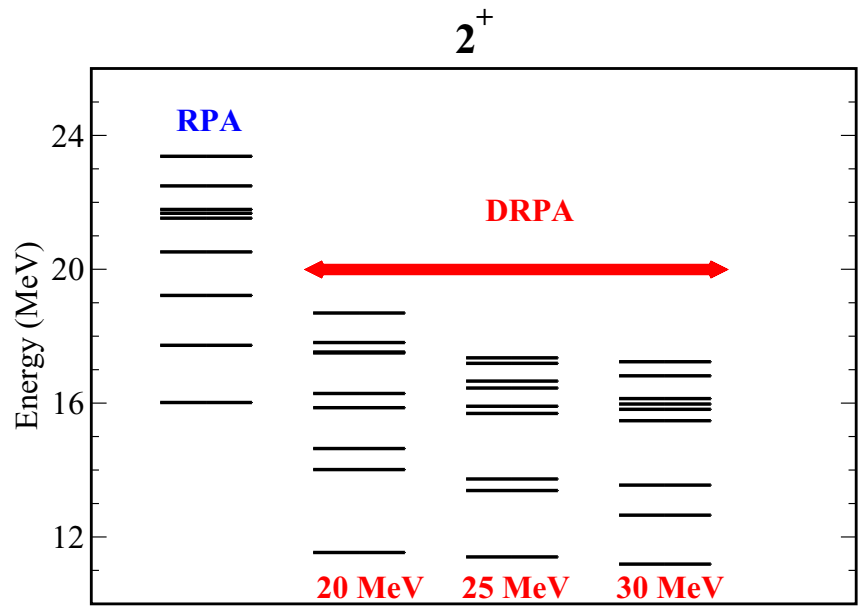

FIG. 3. Same as in Fig. 1, but for the quadrupole states.

$d_{v_{1}^{\prime} v_{2}^{\prime}}^{\alpha^{\prime}} \sum_{\alpha} T_{0 \alpha}$ on Eq. (19), and summing side by side the obtained equations we get the EWSR

$$
\frac{1}{2}\langle H F|[T,[H, T]]| H F\rangle=\sum_{\alpha} E_{\alpha}\left|T_{0 \alpha}\right|^{2} .
$$

We also note that, since the transition amplitudes are evaluated, as in RPA, with reference to the HF ground state, one has

$T_{0 \alpha} \approx\left\langle H F\left|\left[\Lambda_{\alpha}, T\right]\right| H F\right\rangle=\sum_{\nu} c_{\nu}^{\alpha} \sum_{p h}\left(X_{p h}^{v}+Y_{p h}^{v}\right)(p|T| h)$.

In deriving the last equation it has been assumed that $T$ is a one-body operator.

\section{RESULTS FOR ${ }^{16} \mathrm{O}$}

We have applied the DRPA method to the study of the excitation spectrum in ${ }^{16} \mathrm{O}$. In this section we present the results of these calculations and compare them with those obtained in Ref. [14] within the SRPA. The latter were shown

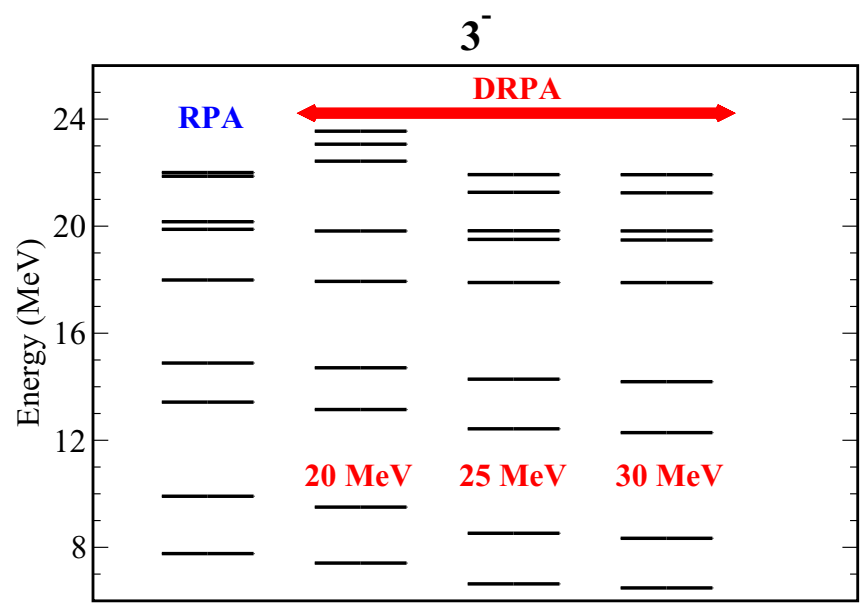

FIG. 4. Same as in Fig. 1, but for the octupole states. 


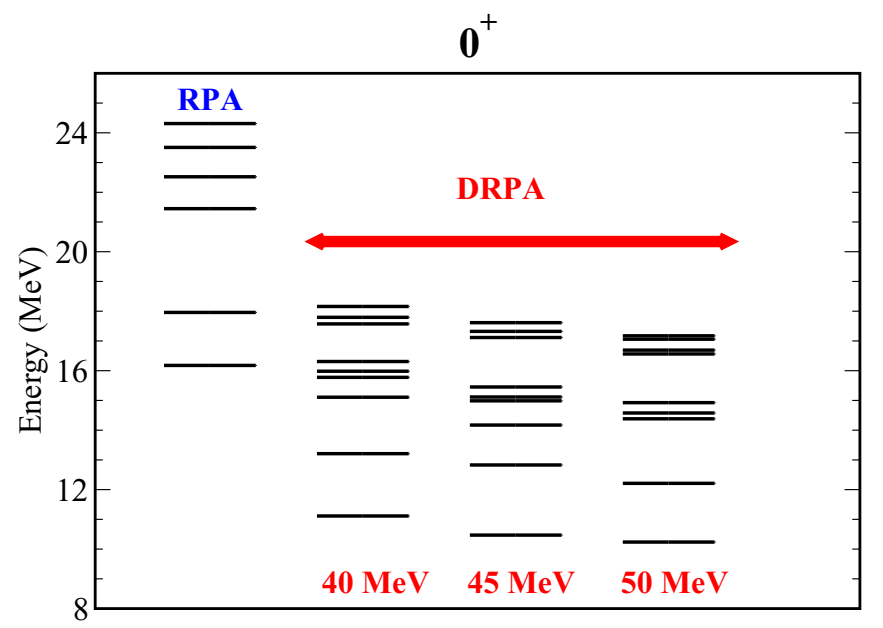

FIG. 5. Monopole states calculated with the RPA and the DRPA, with three different values of the cutoff $E 2 C$, from 40 to $50 \mathrm{MeV}$ and a value of $30 \mathrm{MeV}$ for the $E 1 C$ cutoff.

to be very different from the RPA ones, also in the parts of the spectrum which are expected to be reasonably well described by RPA. As in Ref. [14], all calculations have been done by using the SGII Skyrme interaction [38]. Starting with a HF calculation, natural-parity RPA elementary excitations are obtained including $1 \mathrm{p}-1 \mathrm{~h}$ configurations with unperturbed energy up to $60 \mathrm{MeV}$. These RPA phonons are then used as building blocks to construct a basis of one- and two-phonon states with RPA energies up to some given cutoffs, $E 1 C$ and $E 2 C$, respectively. In order to avoid any misunderstanding due to the use of the word phonon, we stress again that we do not make any bosonic mapping of the RPA elementary excitations, that is, the fermionic internal structure is maintained and the Pauli principle is fully preserved. First of all, it is necessary to assess the stability of the results with respect to the values of the cutoffs $E 1 C$ and $E 2 C$. In Figs. 1-4 we show the energies obtained with a fixed value of $E 2 C=40 \mathrm{MeV}$, with values of $E 1 C$ running from 20 to $30 \mathrm{MeV}$, for the multipole states $0^{+}$, $1^{-}, 2^{+}$, and $3^{-}$. We report also the RPA energies. In all cases, as expected, we see that the energies obtained by diagonalizing

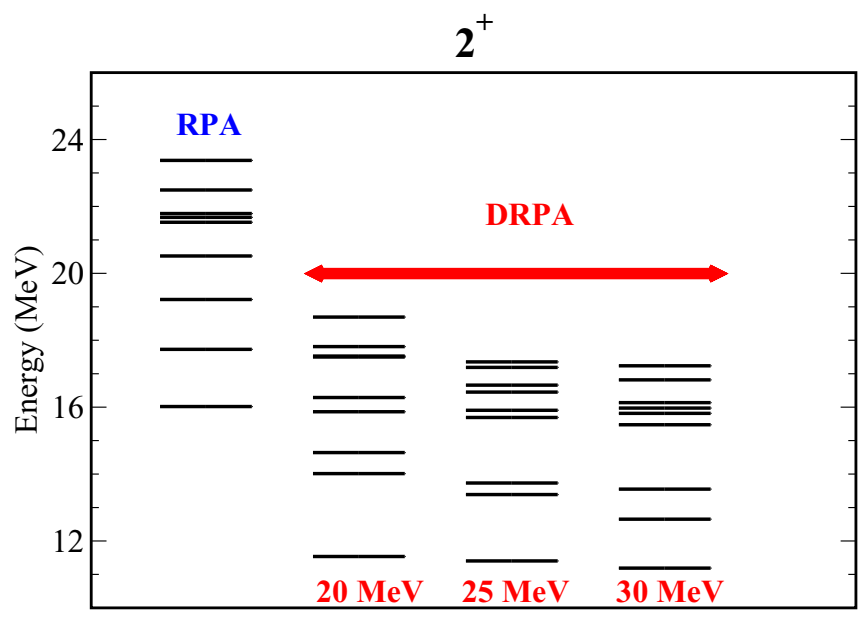

FIG. 7. Same as in Fig. 5, but for the quadrupole states.

the Hamiltonian in the space of one and two RPA phonons are lower than the RPA results. In the cases of positive-parity states this lowering is very pronounced. This happens because, for the nucleus we are dealing with, $1 \mathrm{p}-1 \mathrm{~h}$ configurations of positive parity correspond to a two-major-shell jump and the corresponding unperturbed energies are quite large. The lower-energy states found with the DRPA in the $0^{+}$and $2^{+}$cases have mostly a $2 \mathrm{p}-2 \mathrm{~h}$ nature, as was shown with genuine SRPA calculations [27] and cannot thus be found with the RPA. They can be predicted here by coupling low-lying negative-parity RPA phonons. However, for the moment, we want to stress that for all multipolarities the results stabilize with increasing $E 1 C$ from 20 to $30 \mathrm{MeV}$. To complete this analysis on the stability of our calculations, in Figs. 5-8 we show the results obtained by fixing $E 1 C$ to the value of $30 \mathrm{MeV}$ and varying $E 2 C$ from 40 to $50 \mathrm{MeV}$. Again, the results are remarkably stable.

We stress that, due to the Pauli principle, the set of twophonon states is redundant. This means that the associated generalized eigenvalue problem may present unphysical solutions if the norm matrix presents singularities. The solution of the eigenvalue problem is achieved by using a $Q Z$ algorithm [39].

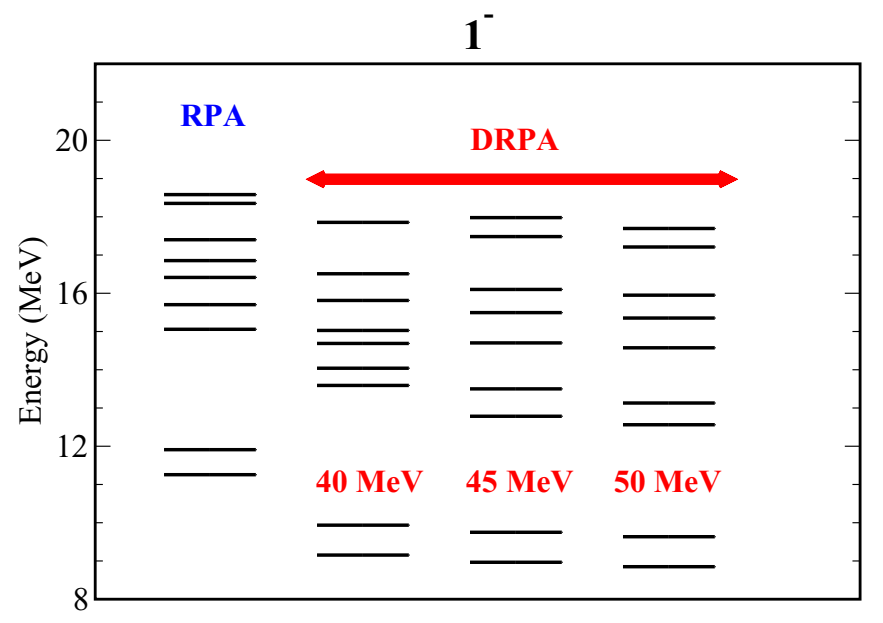

FIG. 6. Same as in Fig. 5, but for the dipole states.

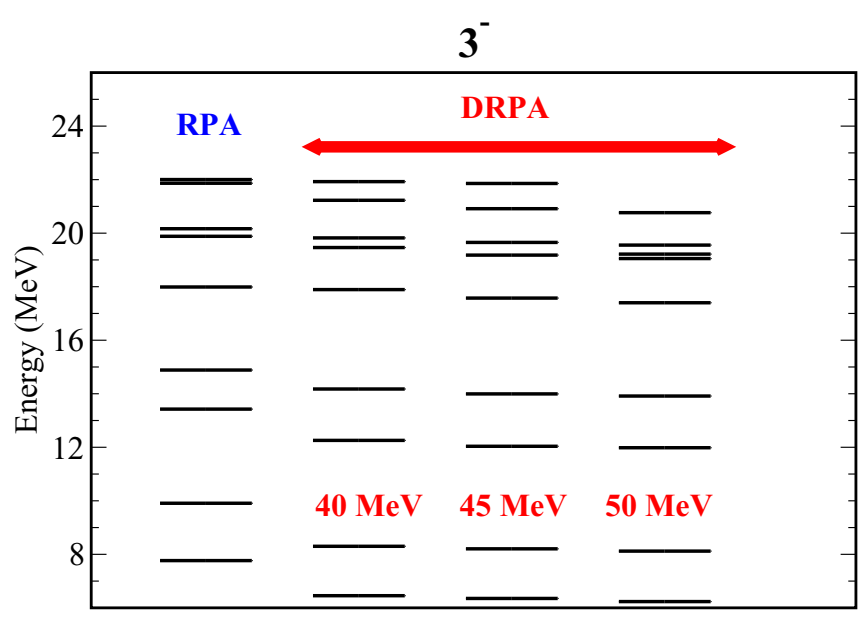

FIG. 8. Same as in Fig. 5, but for the octupole states. 
$\mathrm{J}^{\pi}=0^{+}, \mathrm{T}=0$

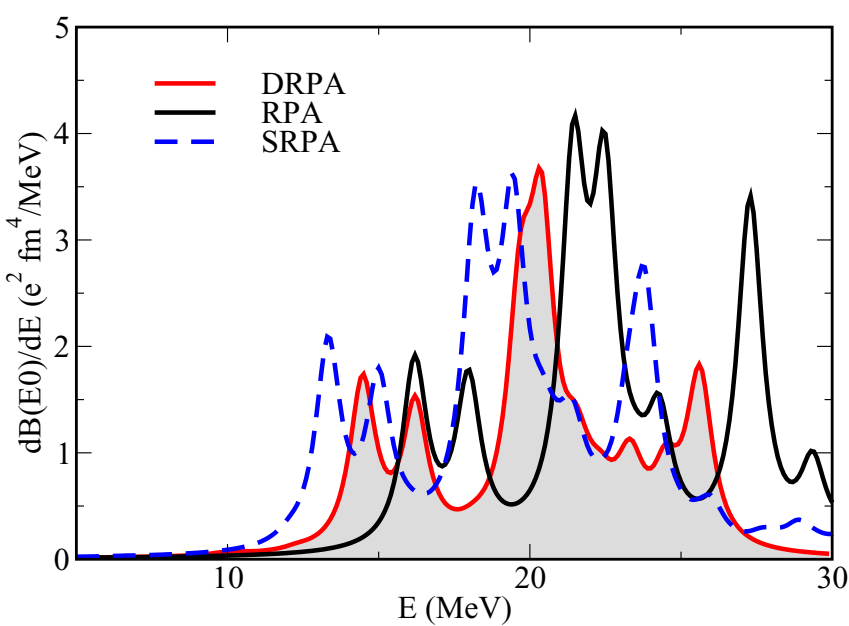

FIG. 9. Monopole response obtained with the RPA (black solid line), the SRPA (blue dashed line), and the DRPA (red solid line and grey area).

The employed algorithm provides the physical eigenvalue as the ratio of two quantities $\alpha$ and $\beta$, the latter approaching zero, in the case of singularities of the norm matrix. By analyzing the $\beta$ quantities, we can identify and isolate spurious states associated to the redundancy. Moreover, we notice that, in general, two different groups of eigenvalues are found. A first class, for which the corresponding $\beta$ value is quite stable with respect to the increasing of the model space (that is, the single-particle basis, and the $E 1 C$ and $E 2 C$ cutoff values), and a second one exhibiting instead a quite strong dependence on the model space. Such states have $\beta$ values that are typically much smaller than those of the states belonging to the first group. In practice, for each solution, by increasing the model space dimension (in particular with respect to the $E 2 C$ cutoff), we studied carefully the behavior of the $\beta$ values and only those eigenvalues characterized by stable values have been retained.

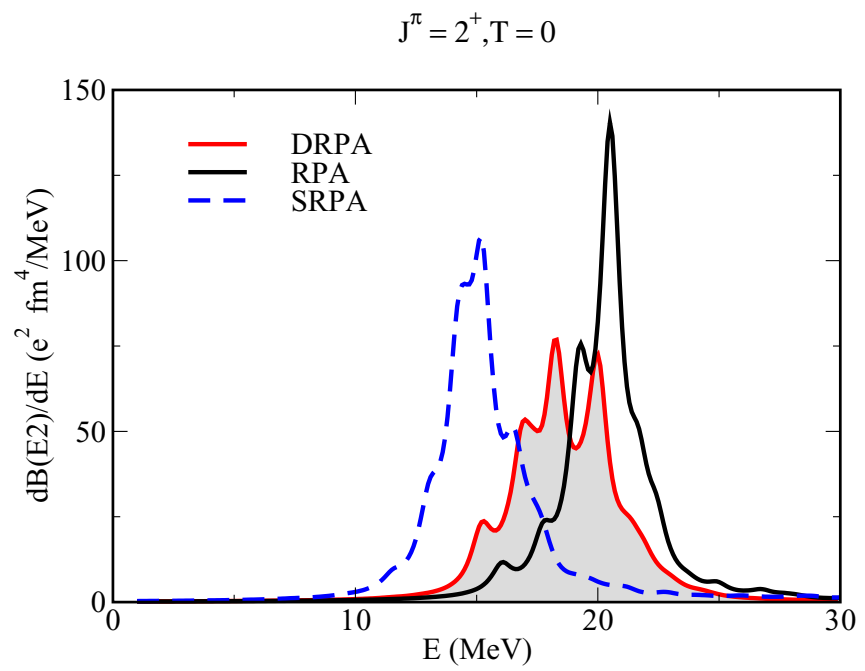

FIG. 11. Same as in Fig. 9, but for the isoscalar quadrupole response.

Let us now turn our attention to the multipole strength distributions. The results within the present approach, with $E 1 C=30 \mathrm{MeV}$ and $E 2 C=50 \mathrm{MeV}$, are presented in Figs. 9-12. The shown curves have been obtained by folding the discrete spectrum with a Lorentzian having a $1 \mathrm{MeV}$ width. In the same figures the RPA and SRPA results are shown.

Due to the one-body nature of the transition operators, the states having a vanishingly small one-phonon component cannot be excited from the ground state. This is the reason why almost no strength is seen below $\sim 12 \mathrm{MeV}$ in the DRPA response for the $0^{+}$and $2^{+}$cases. As a general trend, we see in all cases a shift of the DRPA strength distribution with respect to the RPA, but much less pronounced than in the SRPA. This is a quite satisfactory result because the positions of the main peaks of the isovector and isoscalar GR remain not much lower than in the RPA, and it is known that these excitations are in general well described by the RPA model. Our present results

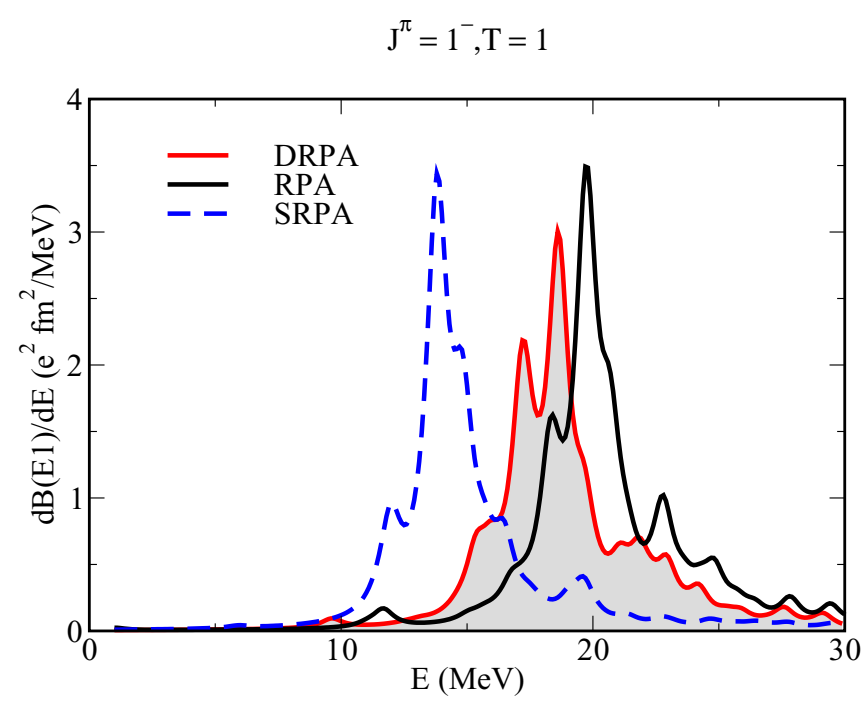

FIG. 10. Same as in Fig. 9, but for the dipole response.

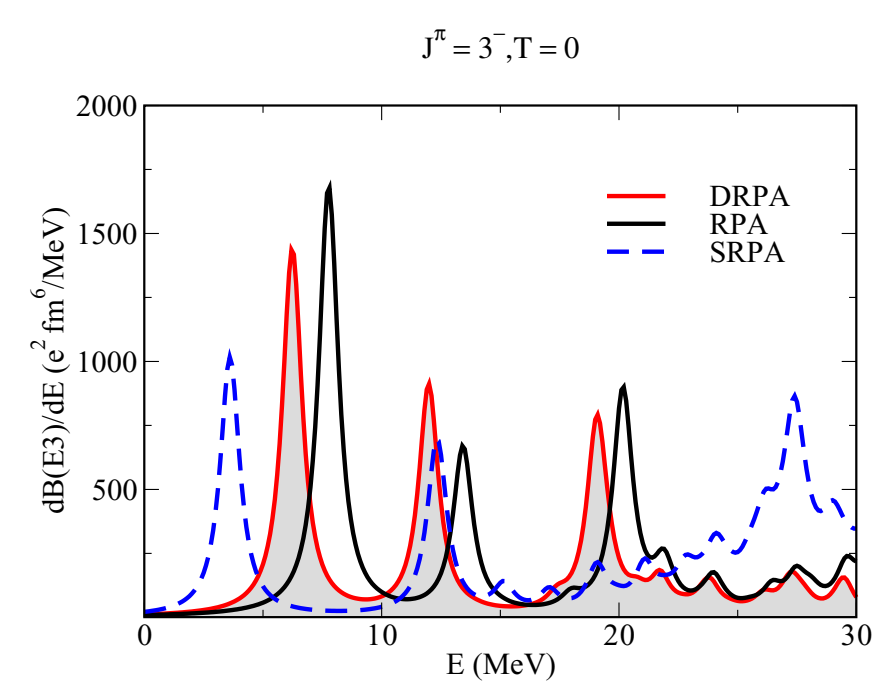

FIG. 12. Same as in Fig. 9, but for the isoscalar octupole response. 

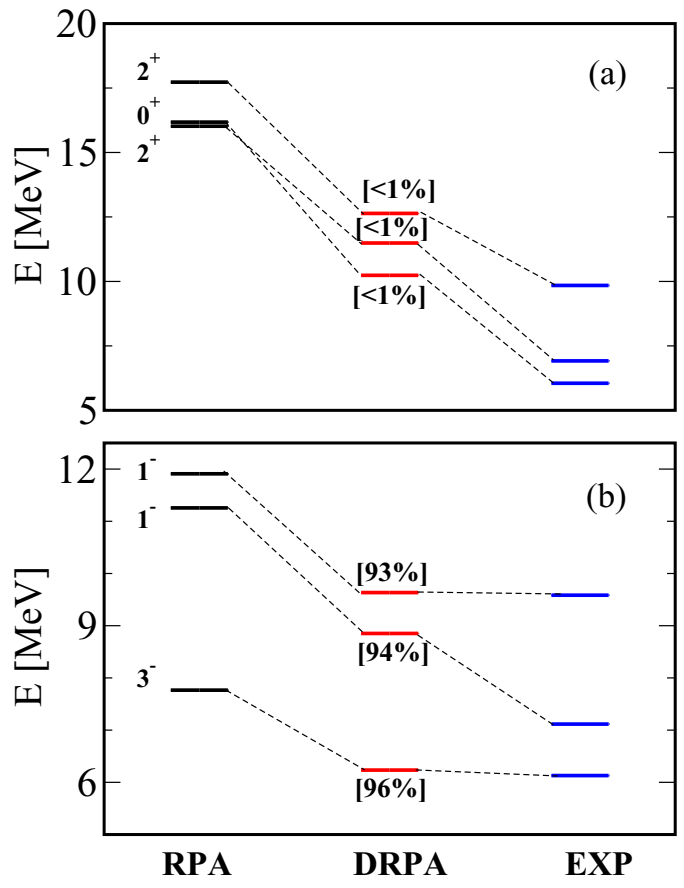

FIG. 13. Comparison of the positive-parity (upper panel) and negative-parity (lower panel) low-lying states obtained in RPA and DRPA with the experimental ones. In the DRPA case, the values in brackets indicate the corresponding one-phonon percentage in the wave functions.

show a shift, with respect to the RPA energies, that is similar to that found with the subtracted SRPA scheme of Ref. [27], and that provided a satisfactory agreement with the corresponding experimental responses [40].

In particular, the lowest $3^{-}$level is predicted at an energy very close to the experimental value [41]. In Fig. 12 we see that the SRPA response shows a wide peak centered around $27 \mathrm{Mev}$, which is not present in RPA and DRPA. This comes from some RPA high-lying energy strength that is shifted downward in SRPA.

In Fig. 13 we show some positive (upper panel) and negative (lower panel) parity low-lying levels obtained in DRPA and the corresponding experimental ones [41]. The numbers in brackets indicate their one-phonon content (and equivalently the corresponding $1 \mathrm{p}-1 \mathrm{~h}$ one). The RPA energies are also reported. For the negative-parity states the agreement of the DRPA positions with the experimental values is fairly good and the corrections to RPA quite important. On the contrary, for the positive-parity states despite the large lowering from the RPA energies, the DRPA values remain still quite different from the experimental ones. This is an indication of the need to include explicitly at least $2 \mathrm{p}-2 \mathrm{~h}$ configurations besides those already taken into account within the DRPA through the action of one and two RPA phonons on the ground state. Concerning the transition probabilities and their comparison with experimental data, we found the following results. For negative-parity states, mainly one-phonon states, the DRPA results are not very different from the RPA ones. For example, for the $3^{-}$low-lying state, the $B(E 3 \uparrow)$ is $721 \mathrm{e}^{2} \mathrm{fm}^{6}$ in RPA, in agreeement with other RPA calculations (see, for example, Refs. [42,43]). In DRPA, as a consequence of the coupling with two-phonon states, the $B(E 3 \uparrow)$ is lowered to $610 \mathrm{e}^{2} \mathrm{fm}^{6}$, while experimentally a value of $1480 \mathrm{e}^{2} \mathrm{fm}^{6}$ is found [44]. For the two $1^{-}$low-lying states, the RPA transition probabilities strongly overestimate the experimental values and the DRPA is not able to correct them significantly. A different scenario is found instead for the positive-parity states, that, as mentioned above, are essentially two-phonon states in DRPA. Due to the use of a pure one-body transition operator, the DRPA transition probabilities are much smaller than the experimental values. In this case, a more general transition operator, containing also a two-body part, as done for example in Ref. [16], could be more suitable.

\section{CONCLUSIONS}

We have presented an extension of the RPA model, denoted here as DRPA, allowing us to include approximately $2 \mathrm{p}-2 \mathrm{~h}$ configurations, in a less explicit way compared to the SRPA model. The DRPA uses RPA phonons (up to two) as building blocks to construct the excited states.

We have presented an application to low-lying states and giant resonances in the nucleus ${ }^{16} \mathrm{O}$.

In the case of giant resonances, the comparison with the RPA and the SRPA results indicates that (i) there is a global downwards shift of the energies with respect to the RPA spectra and (ii) this shift is, however, strongly reduced with respect to that produced by the standard SRPA. The DRPA results are in reasonably good agreement with the experimental responses.

This method represents for these cases an alternative way to include multiparticle-multihole configurations and to correct, at the same time, for the anomalous shift generated by the SRPA. Also for the low-lying states there is a global downwards shift with respect to the RPA results. The $1^{-}$and $3^{-}$ states are in good agreement with the experimental values. The $0^{+}$and $2^{+}$states, however, have larger energies with respect to the measured values, even if the correction with respect to the RPA goes in the good direction, lowering the energies. This discrepancy with the experimental values may be understood as due to the almost pure $2 \mathrm{p}-2 \mathrm{~h}$ nature of the low-lying $0^{+}$ and $2^{+}$states. The implicit inclusion of $2 \mathrm{p}-2 \mathrm{~h}$ configurations induced by the DRPA is not enough to well describe these excitations, and higher-order calculations would be necessary.

\section{ACKNOWLEDGMENTS}

M.V.A. acknowledges the financial support provided by the Ministerio de Economía y Competitividad (FIS2013-41994P, FIS2011-28738-c02-01) and by Consejería de Economía, Innovación, Ciencia y Empleo, Junta de Andalucía (FQM-160, P11-FQM-7632). 
[1] A. Bohr and B. R. Mottelson, Nuclear Structure, Vol. II (Benjamin, New York, 1975).

[2] M. N. Harakeh and A. Van der Woude, Giant Resonances (Clarendon, Oxford, UK, 2001).

[3] W. A. de Heer, Rev. Mod. Phys. 65, 611 (1993).

[4] M. Brack, Rev. Mod. Phys. 65, 677 (1993).

[5] D. J. Rowe, Nuclear Collective Motion (Methuen, London, 1970).

[6] P. Ring and P. Schuck, The Nuclear Many-Body Problem (Springer, New York, 1980).

[7] D. J. Thouless, Nucl. Phys. 21, 225 (1960).

[8] M. Brenna, G. Colò, and P. F. Bortignon, Phys. Rev. C 85, 014305 (2012).

[9] N. Lo Iudice, V. Yu Ponomarev, Ch. Stoyanov, A. V. Sushkov, and V. V. Voronov, J. Phys. G39, 043101 (2012).

[10] E. G. Lanza, F. Catara, M. V. Andrés, Ph. Chomaz, M. Fallot, and J. A. Scarpaci, Phys. Rev. C 74, 064614 (2006).

[11] C. Yannouleas, Phys. Rev. C 35, 1159 (1987).

[12] S. Adachi and E. Lipparini, Nucl. Phys. A 489, 445 (1988).

[13] P. Papakonstantinou, Phys. Rev. C 90, 024305 (2014).

[14] D. Gambacurta, M. Grasso, and F. Catara, Phys. Rev. C 81, 054312 (2010).

[15] D. Gambacurta, M. Grasso, V. De Donno, G. Co', and F. Catara, Phys. Rev. C 86, 021304(R) (2012).

[16] P. Papakonstantinou and R. Roth, Phys. Lett. B 671, 356 (2009); Phys. Rev. C 81, 024317 (2010).

[17] D. Gambacurta and F. Catara, Phys. Rev. B 79, 085403 (2009).

[18] C. Yannouleas, F. Catara, and N. Van Giai, Phys. Rev. B 51, 4569 (1995).

[19] L. G. Gerchikov, C. Guet, and A. N. Ipatov, Phys. Rev. A 66, 053202 (2002).

[20] S. Drozdz et al., Phys. Rep. 197, 1 (1990).

[21] G. Lauritsch and P. G. Reinhard, Nucl. Phys. A 509, 287 (1990).

[22] K. Takayanagy, K. Shimizu, and A. Arima, Nucl. Phys. A 477, 205 (1988).

[23] A. Mariano, F. Krmpotić, and A. F. R. de Toledo Piza, Phys. Rev. C 49, 2824 (1994).
[24] D. J. Thouless, Nucl. Phys. 22, 78 (1961).

[25] V. I. Tselyaev, Phys. Rev. C 75, 024306 (2007).

[26] V. I. Tselyaev, Phys. Rev. C 88, 054301 (2013).

[27] D. Gambacurta, M. Grasso, and J. Engel, Phys. Rev. C 92, 034303 (2015).

[28] D. Gambacurta, M. Grasso, F. Catara, and M. Sambataro, Phys. Rev. C 73, 024319 (2006).

[29] D. Gambacurta and F. Catara, Phys. Rev. B 81, 085418 (2010).

[30] G. Colò, H. Sagawa, and P. F. Bortignon, Phys. Rev. C 82, 064307 (2010).

[31] A. Klein and E. R. Marshalek, Rev. Mod. Phys. 63, 375 (1991).

[32] M. Grasso, F. Catara, and M. Sambataro, Phys. Rev. C 66, 064303 (2002).

[33] F. Andreozzi, F. Knapp, N. Lo Iudice, A. Porrino, and J. Kvasil, Phys. Rev. C 78, 054308 (2008).

[34] F. Knapp, N. Lo Iudice, P. Veselý, F. Andreozzi, G. De Gregorio, and A. Porrino, Phys. Rev. C 90, 014310 (2014).

[35] V. G. Soloviev, Theory of Atomic Nuclei: Quasiparticles and Phonons (Institute of Physics, Bristol, 1992).

[36] D. Gambacurta, M. Grasso, and F. Catara, J. Phys. G 38, 035103 (2011).

[37] S. Y. Li, A. Klein, and R. M. Dreizler, J. Math. Phys. 11, 975 (1970).

[38] N. Van Giai and H. Sagawa, Phys. Lett. B 106, 379 (1981); Nucl. Phys. A 371, 1 (1981).

[39] G. H. Golub and C. Van Loan, Matrix Computations (John Hopkins University Press, Baltimore, 1996); C. B. Moler and G. W. Stewart, SIAM J. Num. Anal. 10, 241 (1973).

[40] Y.-W. Lui, H. L. Clark, and D. H. Youngblood, Phys. Rev. C 64, 064308 (2001).

[41] D. R. Tilley, H. R. Weller, and C. M. Cheves, Nucl. Phys. A 564, 1 (1993).

[42] J. P. Blaizot and D. Gogny, Nucl. Phys. A 284, 429 (1977)

[43] H. Imagawa and Y. Hashimoto, Phys. Rev. C 67, 037302 (2003).

[44] T. Kibédi and R. H. Spear, At. Data Nucl. Data Tables 80, 35 (2002). 